\title{
SURVIVAL TIME OF HIV-INFECTED CHILDREN UNDER 15 YEARS OF AGE AFTER INITIATION OF ANTIRETROVIRAL THERAPY IN GONDAR UNIVERSITY HOSPITAL, ETHIOPIA
}

\author{
ASRAT ATSEDEWEYN and YEMANE ASMELASH \\ Department of Epidemiology and Biostatistics \\ University of Gondar \\ Ethiopia \\ e-mail: asrat07@gmail.com \\ Department of Statistics \\ Aksum University \\ Ethiopia \\ e-mail: yemu9368@gmail.com
}

\begin{abstract}
HIV has emerged as one of the leading causes of childhood mortality and morbidity in sub-Saharan Africa. But, the attention given to HIV-infected children in terms of providing ART had so far taken a second rank. The study had the objectives to identify predictors that have significant impact on the survival status of HIV infected children who received antiretroviral treatment care in Gondar University Hospital, Gondar, Ethiopia. The data used in the study was based on secondary data from hospital records of HIV infected children aged below 15 years who started ART between 2008 and 2013 were included and followed until April 2015 in Gondar University Hospital, Gondar, Ethiopia. Multivariable Cox proportional model was fitted to identify factors
\end{abstract} 2010 Mathematics Subject Classification: 92B15.

Keywords and phrases: children, antiretroviral therapy (ART), HIV, survival, Ethiopia. Received July 19, 2017

(C) 2017 Scientific Advances Publishers 
affecting survival of children after initiation of ART. The median survival time was found to be 55 months. At the end of the follow up, 46 (17.1\%) children died due to the disease, the remaining $223(82.9 \%)$ were alive and loss to follow-up. The multivariate analysis of Cox regression model gives that age of a patient (for age < 1.5 years HR: 3.590; 95\% CI: 1.439, 8.953; $\mathrm{P}=0.006$ ), baseline hemoglobin level (for hemoglobin level < 7g/dl HR: 6.286; 95\% CI: 2.328, 16.973; $\mathrm{P}=0.000$ ), WHO clinical stage (for stage III HR: 0.308; 95\% CI: 0.150, 0.630;

$\mathrm{P}=0.001$ ) and baseline CD4 count (HR: 0.180; 95\% CI: 0.084, 0.388; $\mathrm{P}=0.000$ ) are significant risk factors of survival of HIV infected children during the 92 months of follow up. Therefore, special attention should be given to younger children in ART; patients with low CD4 cell count, patients with advanced WHO clinical staging (stage III and IV) and patients with low hemoglobin level are necessary in order to improve the survival of HIV infected children treated with ART.

\section{Introduction}

For three decades, HIV/AIDS has been overwhelming individuals and families with the disaster of untimely death and medical, financial and social burdens. The occurrences of AIDS epidemics are amongst the forefront public health challenges that the world has faced in recent past [1].

HIV/AIDS have been strong emotional effects on individuals and families with the disaster of untimely death and medical, financial and social burdens for the past three decades. The occurrence of AIDS epidemics are amongst the forefront public health challenges that the world has faced in recent past. Millions of people died of HIV infection during the last three decades. Globally, an estimated 35 million (33.2 million-37.2 million) people were living with HIV in 2013 [2]. In subSaharan Africa, the number of AIDS-related deaths fell by $39 \%$ between 2005 and 2013. The region still accounted for $74 \%$ of all the people dying from AIDS-related causes in 2013 [1]. There are 2.9 million (2.6 million -3.2 million) children (aged 0-14) living with HIV in sub-Saharan Africa. Of the estimated 1.8 million people living with HIV 1.5 million were living in this area. There were also 190,000 children died of AIDS-related illnesses during 2013, out of 1.5 million people overall [3]. 
Ethiopia is one of the sub-Saharan African countries hardly-hit by HIV/AIDS in all its manifestations. In 2013, there were an estimated 793,700 (716,300-893,200) people living with HIV including 200,300 (172,400-232,400) children according to the latest EPP/Spectrum modelling [4].

Treatment of HIV- infected children with antiretroviral therapy (ART) leads to immune reconstitution as shown by increase in CD4 lymphocyte counts, decreased risk of opportunistic infection and improved survival [1, 2]. Moreover, children may die with undetectable viral load and adequate CD4 count recovery [4]. However, very little attention has been given to look into how factors other than ART drugs may influence the survival of HIV infected children. Therefore, this research work is undertaken against the above background and explores the factors that have strong association with the survival experience of HIV-infected children treated with ART in Gondar University Hospital.

The study had the objectives to assess the relationship of explanatory variables to survival time, estimate the survival duration and identify predictors that have significant impact on the survival status of HIV infected children who received antiretroviral treatment and care in Gondar University Hospital, Gondar, Ethiopia.

\section{Methods}

The data for the study were obtained from patients' follow up records of a total of 756 children on ART follow ups during the study period from the HIV cohort database. However, only 269 patients with a full record of variables who started ART between 2008 and 2013 were included and followed until April 2015 in Gondar University Hospital.

The study focused only on demographic variables (age, gender) and clinical/immunological variables (baseline weight, WHO clinical stage, prophylaxis taken, baseline functional status, baseline TB status, reason for taking ART, baseline CD4 count, total lymphocytes counts, baseline 
hemoglobin levels, opportunistic illness, type of ART drug) that can affect the survival time of HIV-infected children.

The response variable in the study is the survival time of HIVinfected children measured in months after starting ART. This is measured as the time a child was followed up from the time the child began to receive treatment, until the time to an event (death) or they were lost to follow up (for those right censored subjects).

\section{Statistical Model}

A variety of models and methods have been developed for doing this sort of survival analysis using either parametric or semi-parametric approaches. One of the most popular types of regression models used in survival analysis is the Cox proportional hazard model [7].

The Cox regression model is used to determine which combinations of explanatory variables affect the form of the hazard function. Also the model is used to obtain an estimate of the hazard function itself for an individual who may be of interest in its own right.

The Cox regression model can be used for data that contain censored observations. The model also takes into account the fact that the probability of experiencing an event differs with duration of exposure to risk. In particular, we apply semi-parametric Cox proportional hazards model, it is the most commonly used model in hazard regression.

\section{Results}

A total of 269 participants with full record of variables were included in the study, from this $46(17.1 \%)$ children died due to the disease, 223 (82.9\%) were dead and loss to follow-up during the time of data collection. Out of the total 269 ART followers, 116 (36.65\%) were male and the rest were females. Among 269 children, 78 (30\%) were at clinical stage IV, 82 (30.5\%) were at clinical stage III, 63 (23.4\%) were at clinical stage II and 
the rest $46(17.1 \%)$ were at clinical stage I when they started ART (see Table 4.1).

Table 4.1. Distribution of important socio-demographic and health factors of HIV infected children

\begin{tabular}{|c|c|c|c|c|}
\hline Covariates & Category & Censored (\%) & Dead & Total \\
\hline \multirow[t]{3}{*}{ Age } & Age less than 1.5 years & $19(67.9 \%)$ & 19 & 28 \\
\hline & Ages between $1.5-5$ years & $75(78.1 \%)$ & 21 & 96 \\
\hline & Ages between $5-14$ years & $129(89.0 \%)$ & 16 & 145 \\
\hline \multirow[t]{2}{*}{ Gender } & Male & $92(79.3 \%)$ & 24 & 116 \\
\hline & Female & $131(85.6 \%)$ & 22 & 153 \\
\hline Baseline & Working & $124(87.3 \%)$ & 18 & 142 \\
\hline \multirow[t]{2}{*}{ Functional Status } & Ambulatory & $58(85.3 \%)$ & 10 & 68 \\
\hline & Bedridden & $41(69.5 \%)$ & 18 & 59 \\
\hline \multirow[t]{4}{*}{ Baseline WHO Stage } & Stage-1 & $44(95.7 \%)$ & 2 & 46 \\
\hline & Stage-2 & $59(95.2 \%)$ & 3 & 62 \\
\hline & Stage-3 & $72(86.7 \%)$ & 11 & 83 \\
\hline & Stage- 4 & $48(61.5 \%)$ & 30 & 78 \\
\hline Baseline CD4 & Above the threshold & $120(93.0 \%)$ & 9 & 129 \\
\hline Count & Below the threshold & $103(73.6 \%)$ & 37 & 140 \\
\hline \multirow[t]{5}{*}{ Original Regimen } & D4t-3TC-NVP & $85(81.7 \%)$ & 19 & 104 \\
\hline & D4t-3TC-EFV & $8(72.7 \%)$ & 3 & 11 \\
\hline & AZT-3TC-NVP & $102(84.3 \%)$ & 19 & 121 \\
\hline & AZT-3TC-EFV & $9(75.0 \%)$ & 3 & 12 \\
\hline & Other Drugs & $19(90.5 \%)$ & 2 & 21 \\
\hline \multirow[t]{4}{*}{ Reason for ART } & Clinical & $20(87.0 \%)$ & 3 & 23 \\
\hline & CD4 & $87(77.7 \%)$ & 25 & 112 \\
\hline & CD4 \& Clinical & $80(90.9 \%)$ & 8 & 88 \\
\hline & CD4 \& Lymphocytes & $36(78.3 \%)$ & 10 & 46 \\
\hline \multirow[t]{2}{*}{ Prophylaxis use } & No & $68(85.0 \%)$ & 12 & 80 \\
\hline & Yes & $155(82.0 \%)$ & 34 & 189 \\
\hline
\end{tabular}


Table 4.1 (continued)

\begin{tabular}{|l|l|l|l|l|}
\hline Baseline & - ve & $108(84.4 \%)$ & 20 & 128 \\
TB Screen & + ve & $115(81.6 \%)$ & 26 & 141 \\
\hline Opportunistic & No & $102(82.3 \%)$ & 22 & 124 \\
Illness & Yes & $121(83.4 \%)$ & 24 & 145 \\
\hline Baseline & $\leq 7.00 \mathrm{gm} / \mathrm{dl}$ & $76(78.4 \%)$ & 21 & 97 \\
Hemoglobin & $7.00-8.50 \mathrm{gm} / \mathrm{dl}$ & $30(73.2 \%)$ & 11 & 41 \\
level $(\mathrm{gm} / \mathrm{dl})$ & $8.50-10.00 \mathrm{gm} / \mathrm{dl}$ & $43(82.7 \%)$ & 9 & 52 \\
& $>10.00 \mathrm{gm} / \mathrm{dl}$ & $74(93.7 \%)$ & 5 & 79 \\
\hline
\end{tabular}

From results of multivariate analysis using the significant variables in Table 4.2 below, the covariates age, WHO clinical stages, CD4 counts, and hemoglobin level are the four categorical variables that are found to be significantly associated with the survival time of HIV infected children under ART treatment in the fitted Cox regression model.

Table 4.2. Multivariate analysis using the significant variables

Variables in the Equation

\begin{tabular}{|c|c|c|c|c|c|c|c|c|}
\hline & \multirow[t]{2}{*}{ B } & \multirow[t]{2}{*}{$\mathrm{SE}$} & \multirow[t]{2}{*}{ Wald } & \multirow[t]{2}{*}{$\mathrm{df}$} & \multirow[t]{2}{*}{ Sig. } & \multirow[t]{2}{*}{$\operatorname{Exp}(B)$} & \multicolumn{2}{|c|}{$95.0 \%$ CI for $\operatorname{Exp}(B)$} \\
\hline & & & & & & & Lower & Upper \\
\hline Age & & & 11.257 & 2 & 0.004 & & & \\
\hline Age(1) & 1.278 & 0.466 & 7.513 & 1 & 0.006 & 3.590 & 1.439 & 8.953 \\
\hline Age(2) & 0.968 & 0.341 & 8.077 & 1 & 0.004 & 2.632 & 1.350 & 5.130 \\
\hline Baseline WHO Stage & & & 24.502 & 3 & 0.000 & & & \\
\hline Baseline WHO Stage(1) & -2.443 & 0.744 & 10.794 & 1 & 0.001 & 0.087 & 0.020 & 0.373 \\
\hline Baseline WHO Stage(2) & -1.890 & 0.623 & 9.192 & 1 & 0.002 & 0.151 & 0.045 & 0.513 \\
\hline Baseline WHO Stage(3) & -1.179 & 0.366 & 10.370 & 1 & 0.001 & 0.308 & 0.150 & 0.630 \\
\hline Baseline CD4 Count & -1.713 & 0.391 & 19.200 & 1 & 0.000 & 0.180 & 0.084 & 0.388 \\
\hline Baseline Hemoglobin & & & 13.658 & 3 & 0.003 & & & \\
\hline Baseline Hemoglobin(1) & 1.838 & 0.507 & 13.158 & 1 & 0.000 & 6.286 & 2.328 & 16.973 \\
\hline Baseline Hemoglobin(2) & 1.721 & 0.550 & 9.795 & 1 & 0.002 & 5.592 & 1.903 & 16.436 \\
\hline Baseline Hemoglobin(3) & 1.633 & 0.600 & 7.406 & 1 & 0.007 & 5.119 & 1.579 & 16.593 \\
\hline
\end{tabular}


Let us begin with baseline CD4 cell count of the patient that is supposed to be significant both clinically and statistically. In this study, a baseline CD4 cell count has been found to have a significant impact on the survival time of HIV infected children. The estimated hazard ratio for baseline CD 4 counts is 0.180 (with a 95\% CI 0.084-0.388). Thus, patients their CD4 counts are above the threshold levels have $82 \%$ lower risk of death than those that are CD4 counts below the threshold levels. The confidence interval indicates that the risk of death for those patients that have CD 4 counts above the threshold levels could be lower by a quantity as large as $38.8 \%$ or as low as $8.4 \%$ than patients that have CD4 counts below the threshold levels; $p<0.0001$.

HIV-infected children aged below 1.5 years are 3.59 times more likely to die than children aged between 5 to 14 years (the reference category). The 95\% CI confirms that this hazard of death for this category could be as low as 1.439 and as high as 8.953 compared with the reference category. HIV-infected children aged between 1.55 and 5 years are 2.632 times more likely to die than children with age between 5 to 14 years (the reference category). The $95 \%$ CI verifies that the rate of death could be as small as 1.350 and as large as 5.130. HIV-infected children aged below 1.5 years are 1.36 times more likely to die than HIV-infected children aged between 1.5 and 5 years given that all other factors are constant.

The estimated risks of death for a patient with hemoglobin level less than $7 \mathrm{gm} / \mathrm{dl}$ as compared to those patients with hemoglobin level greater than $10 \mathrm{gm} / \mathrm{dl}$ (reference category) are 6.286 (95\% CI: 2.323, 16.973). This means that the hazard rate of death for hemoglobin level less than $7 \mathrm{gm} / \mathrm{dl}$ is 6.286 times more likely to die than HIV-infected children with hemoglobin level greater than $10 \mathrm{gm} / \mathrm{dl}$ (reference category). And also, the estimated relative risk (hazard ratio) of dying for patients with hemoglobin level between $7-8.5 \mathrm{gm} / \mathrm{dl}$ is 5.592 times more likely to die as compared to those patients with hemoglobin level greater than $10 \mathrm{gm} / \mathrm{dl}$ (reference category). The $95 \% \mathrm{CI}$ suggests that the rate of death could be as small as 1.903 and as large as 16.436. Moreover, the estimated hazard 
ratio of hemoglobin level between 8.5 to $10 \mathrm{gm} / \mathrm{dl}$ compared to the reference hemoglobin level is 5.119 (95\% CI: 1.579, 16.593). This implies that the risk dying for a patient with hemoglobin level between 8.5 to $10 \mathrm{gm} / \mathrm{dl}$ is 5.119 more likely to die than those patients with hemoglobin level greater than $10 \mathrm{gm} / \mathrm{dl}$ (reference category). Children with hemoglobin value less than $7 \mathrm{gm} / \mathrm{dl}$ are 1.124 times more likely to die than children with hemoglobin value between $7 \mathrm{gm} / \mathrm{dl}$ to $8.5 \mathrm{gm} / \mathrm{dl}$. Moreover, children with hemoglobin level greater than $10 \mathrm{gm} / \mathrm{dl}$ are $84 \%$ less likely to die than children with hemoglobin value less than $7 \mathrm{gm} / \mathrm{dl}$ provided that all other factors are held constant.

The reference category for the design variables of WHO clinical stage is patients who are in WHO clinical stage IV. The estimated hazard ratio for clinical stage III is 0.308 (with a $95 \%$ CI $0.15-0.630$ ). Thus, a patient whose clinical stage is III has approximately 0.308 times the hazard faced by patient whose clinical stage is IV (or the hazard of death for clinical stage III are $70 \%$ less likely to die than those of clinical stage IV).

On the other hand, the estimated hazard ratio of stage III compared to stage II was $2.04=\exp (-1.179-(-1.890))$. Since the confidence interval does not contain 1, an individual in clinical stage III has a significantly higher hazard rate than patients in clinical stage II. Thus, patients in stage III are 2.04 times more likely to die than patients in stage II.

\section{Assessing the goodness of fit of the model}

We need to check the goodness of fit of the proportional hazards model based on the empirical data. Therefore, for the model fitted in this

study the likelihood ratio, score and Wald tests are used to compare (at $5 \%$ significance level) the goodness of fit of the model.

The SAS output in Table 4.3 reveal that the log partial likelihood function (-2LL) without covariate was 489.281 while the function with 
significant covariates was 410.634 . This result shows that the model is appropriate with Chi-square of 78.647 with 9 degrees of freedom and $p$-value $<0.0001$.

Table 4.3. Likelihood ratio, score and Wald tests for testing the global null hypothesis of $\beta=0$

Testing Global Null Hypothesis: $\beta=0$

\begin{tabular}{|l|c|c|c|}
\hline Test & Chi-square & Df & Pr $>$ Chisq \\
\hline Likelihood ratio & 78.647 & 9 & $<0.0001$ \\
\hline Score & 74.786 & 9 & $<0.0001$ \\
\hline Wald & 67.759 & 9 & $<0.0001$ \\
\hline
\end{tabular}

Moreover, Cox-Snell residuals are used to assess the overall goodness of fit of the model. The plot in Figure 1 of the cumulative hazard function of the Cox-Snell residual against the Cox-Snell residuals are fairly close to the $45^{\circ}$ straight line through the origin. This suggested that the model fit to the data is satisfactory. The $45^{\circ}$-straight line through the origin is drawn for reference line.

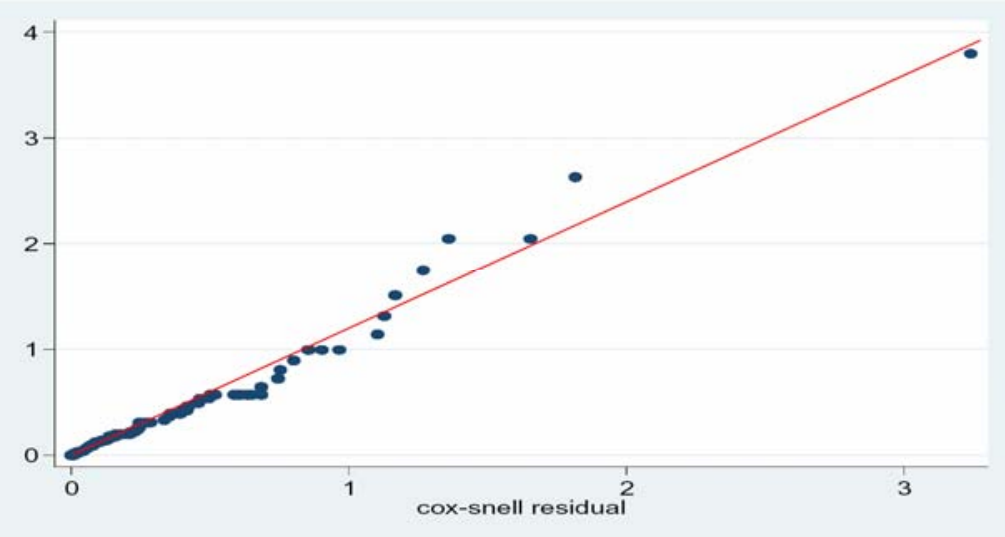

The 45o-striaght line through the origin is drawn for reference line.

Figure 1. Cumulative hazard plot of the Cox Cox-Snell residuals of the Cox proportional hazards regression model. 


\section{Discussion}

This study identified variables/factors that are significantly associated with survival time of HIV infected children under ART treatments. The results of this study show that the risk of death among HIV infected children for the age groups less than 1.5 years and 1.5 to 5 years are higher than those ages between 5 to 14 years. A study by Aregay et al. [9] identifies independent predictors of children's mortality on ART. The result suggested that mortality of children on ART was low and factors that affect mortality of children on ART were age less than 18 months.

A similar study by Mary Munyagwa et al. [8] also found that mortality among HIV-infected children was highest among those aged less than 2 years. Thus, mortality among these high risk groups contributed to the higher rate of mortality.

CD4 cell count is the most important marker of HIV disease progression and a strong predictor of survival HIV-infected children, similar to the plasma viral load. That may be due to the fact that HIV attacks CD4 cells, and as time goes by people with HIV often see their CD4 cell counts drop. That is the lower the CD4 cell count the greater the chances of getting a number of very serious diseases. The significant impact of CD4 cell count on survival rate has been revealed by many studies.

A study in $[10,11,12]$ reported that patients with low baseline CD4 cell count were a strong risk factor for early mortality of HIV-infected children. Our results are also consistent with the findings in the above studies. But, a similar study conducted by Habtamu in Bahir-Dar were found that low baseline CD4 count was not predictor of survival time of HIV infected children [13]. His result contradicts with our findings that low baseline CD4 cell count were a strong risk factor for survival time of HIV infected children. 
The finding of this study tells that observed higher risk of dying was found among HIV-infected children with lower hemoglobin level (anemic groups) compared to hemoglobin level greater than $10 \mathrm{gm} / \mathrm{dl}$, this study was consistent with other studies conducted elsewhere. According to Getachew et al. [14], it was found that hemoglobin level less than $7 \mathrm{gm} / \mathrm{dl}$ were significant independent predictors of death after controlling for other factors. For instance, another study also identifies that the determinants of mortality in Bahir-Dar showed that the risk death is higher among HIV-infected children with a lower hemoglobin level [13]. Therefore, the above studies confirm the same conclusion like our result.

Like CD4 cell count, the WHO clinical staging system has been shown to be a practical and accurate way to manage HIV-infected patients. In this study, we found that the advanced WHO clinical stages III and IV were independent markers of mortality for patients on ART. The possible justification for the finding is that the advanced clinical stage of the disease is the cause for HIV-associated complications. A study in [11] was found that those children with WHO clinical stage IV had an increased risk of death. Similar to our finding, studies by [13, 14, 15] provided evidence that children in advanced clinical stages (III and IV) had a strong association with high mortality of an HIV infected children on ART.

The above sources showed that the most significant predictors of survival of children were CD4 count, advanced WHO clinical stages, age, weight and to some extent opportunistic diseases like anemia and pneumonia. The findings of the current study identified advanced WHO clinical stage, age, hemoglobin level and baseline CD4 count as determinant predictors of survival of HIV-infected children treated with ART at Gondar University Hospital. Weight did not come out as a strong predictor although it is a clinically meaningful determining variable. 


\section{Conclusion}

In this study, we tried to identify the factors that are associated with survival time of HIV infected children treated with ART in Gondar University Hospital using the methods of survival analysis. The KaplanMeier and log-rank test are used to estimate and compare the survival time of children after initiation of ART treatment. The study has shown that the overall median survival time of HIV infected children under the study was 51.1 months. During the follow-up period out $269 \mathrm{HIV}$ infected children 46 (17.1\%) of them experience the event (i.e., dead).

Moreover, the results of the multivariable proportional hazards Cox regression model showed that $\mathrm{CD} 4$ count at the start of ART, age, advanced WHO clinical stages and low hemoglobin level (less than $7 \mathrm{gm} / \mathrm{dl}$ and between 7 to $8.5 \mathrm{gm} / \mathrm{dl}$ ) are associated with higher risk of mortality. And also children whose age less than 1.5 years and age between 1.5 to 5 years and being in advanced WHO clinical stage III \& IV are also associated with increased rate of mortality in both the univariable and multivariable analysis. Similarly, patients with poor health indicators like low baseline CD4 count and low hemoglobin level are less likely to survive.

Therefore, special attention should be given to younger children in ART; children should start ART treatment at early age, with CD4 count at the normal level or above the threshold level, when they have higher hemoglobin values and when they are at a lower clinical stage.

\section{Declarations}

\section{List of Abbreviations}

$3 \mathrm{TC}$

Lamivudine

AIDS Acquired Immunodeficiency Syndrome

ART Antiretroviral therapy

AZT Zidovudine 


$\begin{array}{ll}\text { D4T } & \text { Stavudine } \\ \text { DOTS } & \text { Directly Observed Treatment, Short-course } \\ \text { EDHS } & \text { Ethiopian Demographic Health Survey } \\ \text { EFV } & \text { Efavirenz } \\ \text { EPP } & \text { Estimation and Project Package } \\ \text { HIV } & \text { Human Immunodeficiency Virus } \\ \text { KM } & \text { Kaplan-Meier } \\ \text { LR } & \text { Likelihood Ratio } \\ \text { NNRTI } & \text { Non-Nucleoside Reverse Transcriptase Inhibitor } \\ \text { NRTI } & \text { Nucleoside Reverse Transcriptase Inhibitor } \\ \text { NVP } & \text { Neverapine } \\ \text { PH } & \text { Proportional Hazard } \\ \text { PLWHA } & \text { People Living with HIV/AIDS } \\ \text { SE } & \text { Standard Error } \\ \text { TB } & \text { Tuberculosis } \\ \text { WHO } & \text { World Health Organization }\end{array}$

\section{Acknowledgements}

The authors would like to acknowledge the support of the University of Gondar teaching hospital staff members for dedicating their time in providing the necessary data. In addition, we would like to acknowledge the University of Gondar for its financial support for the research.

\section{References}

[1] UNAIDS, The Gap Report, 2014.

[2] M. L. Newell, H. Coovadia, M. Cortina-Borja, N. Rollins and P. Gaillard, Mortality of infected and uninfected infants born to HIV-infected mothers in Africa, 2004. 
[3] WHO Report in Partnership with UNICEF and UNAIDS, Global update on Health Sector Response to HIV, 2014.

[4] EPP/Spectrum Guidelines, Estimation and Project Package for HIV, 2014.

[5] Federal Democratic Republic of Ethiopia Country Progress Report on the HIV Response, 2014.

[6] UNAIDS Global AIDS Response Progress Reporting, 2015.

[7] D. R. Cox, Regression models and life tables (with Discussion), Journal of the Royal Statistical Society; Series B 34 (1972), 187-220.

[8] Mary Munyagwa, Kathy Baisley, Jonathan Levin, Michael Brian, Heiner Grosskurth and Dermot Maher, Mortality of HIV-infected and uninfected children in a longitudinal cohort in rural south-west Uganda, 17(7) (2012), 836-843.

[9] Aregay Gebremedhin, Solomon Gebremariam, Fisaha Haile, Berhe Weldearegawi and Carla Decotelli, Predictors of mortality among HIV infected children on anti-retroviral therapy in Mekelle Hospital, Northern Ethiopia, BMC Public Health 13 (2013), 1047.

[10] M. T. Brady, J. M. Oleske, P. L. Williams, C. Elgie, L. M. Mofenson, W. M. Dankner et al., Declines in mortality rates and changes in Causes of death in HIV-1-infected children during the HAART era, J. Acquir Immune Defic. Syndr. 53(1) (2010), 86-94.

[11] Pagakrong Lumbiganon, Azar Kariminia, Linda Aurpibul et al., Survival of HIVinfected children in Asia-Pacific region, J. Acquir Immune Defic. Syndr. 56(4) (2011), 365-371.

[12] Wanatpreeya Phongsamart, Rawiwan Hansudewechakul, Torsak Bunupuradah Virat et al., Long-term outcomes of HIV-infected children in Thailand, International Journal of Infectious Diseases 22 (2013), 19-24.

[13] Habtamu Atnafu and Eshetu Wencheko, Factors affecting the survival of HIVinfected children after ART initiation in Bahir-Dar, Ethiopia, Ethiop. J. Health Dev. 26(3) (2012), 193-199.

[14] Getachew Ebissa, Negusse Deyessa and Sibhatu Biadgilign, Predictors of early mortality in a cohort of HIV-infected children receiving high active antiretroviral treatment in public hospitals in Ethiopia, AIDS Care: Psychological and Sociomedical Aspects of AIDS/HIV, 2015.

[15] A. A. Kedir, A. Desta and G. Fesseha, Factors affecting survival of HIV positive children taking antiretroviral therapy at Adama Referral Hospital and Medical College, Ethiopia, J. AIDS Clin. Res. 5 (2014), 289. 